\title{
Model-based multirate Kalman filtering approach for optimal two-dimensional signal reconstruction from noisy subband systems
}

\author{
Jiang Qun Ni \\ Ka Leung Ho \\ Kai Wing Tse \\ University of Hong Kong \\ Department of Electrical and Electronic \\ Engineering \\ Pokfulam Road \\ Hong Kong \\ E-mail: jqni@eee.hku.hk
}

\begin{abstract}
Conventional synthesis filters in subband systems lose their optimality when additive noise (due, for example, to signal quantization) disturbs the subband components. The multichannel representation of subband signals is combined with the statistical model of input signal to derive the multirate state-space model for the filter bank system with additive subband noises. Thus the signal reconstruction problem in subband systems can be formulated as the process of optimal state estimation in the equivalent multirate state-space model. Incorporated with the vector dynamical model, a 2-D multirate state-space model suitable for 2-D Kalman filtering is developed. The performance of the proposed 2-D multirate Kalman filter can be further improved through adaptive segmentation of the object plane. The object plane is partitioned into disjoint regions based on their spatial activity, and different vector dynamical models are used to characterize the nonstationary object-plane distributions. Finally, computer simulations with the proposed 2-D multirate Kalman filter give favorable results. () 1998 Society of Photo-Optical Instrumentation Engineers. [S0091-3286(98)01608-0]
\end{abstract}

Subject terms: Kalman filter; perfect reconstruction filter bank; multirate statespace model.

Paper 27057 received May 28, 1997; revised manuscript received Dec. 16, 1997; accepted for publication Feb. 18, 1998.

\section{Introduction}

Multirate signal processing is of interest to a great many researchers due to its suitability in applications such as digital image/speech coding, progressive image transmission, and spectrum analysis. The idea of multirate signal processing is to decompose the original signal into complementary frequency bands and then process them separately in each subband. There has been tremendous progress in the design of decimation and interpolation filters and analysis/synthesis filter banks that allow perfect reconstruction (PR). ${ }^{1}$ The development of filter bank systems with PR involves the removal of three distortions: aliasing distortion (ALD), amplitude distortion (AMD), and phase distortion (PHD). Thus, in their overall performance, filter-bank systems with PR characteristic can be viewed as systems with all-pass and linear phase transfer functions.

The conventional approaches for PR filter-bank systems are based on the assumption that the subband components are free of noise. However, in practical applications with filter-bank systems, the subband components are always contaminated by noise due to the effect of quantization, roundoff, and other corruption, so that perfect reconstruction is no longer possible. Two typical approaches have been developed to tackle the noise effects in designing filter bank systems. The work in Ref. 2 incorporated the quantizer model in the design of synthesis filter. Through the quantizer-model-based synthesis filter, one can cancel all signal-dependent errors in the output of the system. In an alternative approach, ${ }^{3}$ the optimal filter banks are derived on the basis of minimization of the mean squared error (MSE) between the original and the reconstructed signal, considering the statistical characteristic of the input signal and noise.

The goal of this paper is to improve the applicability of filter-bank systems by proposing a multirate Kalman synthesis filter to replace the conventional synthesis filters, with emphasis on 2-D cases. With the input signal embedded in the state vector, the multichannel representation of subband signals is combined with the statistical model of the input signal to derive the multirate state-space model for the filter-bank systems, and the subband noises are assumed to be additive in this model. On the basis of this model, the multirate Kalman filter can be constructed to provide the minimum-variance estimation of the input signal based on observations of noisy subband components.

The concept of multirate Kalman synthesis filtering was first given in Ref. 4. Our work differs from that in Ref. 4 in that the philosophy to derive the state-space model is different and a multirate state-space model suitable for 2-D Kalman filtering is developed. It is well known that Kalman filtering is one-dimensional in nature. To apply this technique, however, the observation vector must be a function of one independent variable, whereas the 2-D subband components are functions of two spatial variables. In our approach, the vector linear dynamical model developed in Ref. 5 is incorporated with the 2-D multichannel representations of subband signals and thus results in the final 2-D multirate state-space model for optimal 2-D signal reconstruction from noisy subband systems. 


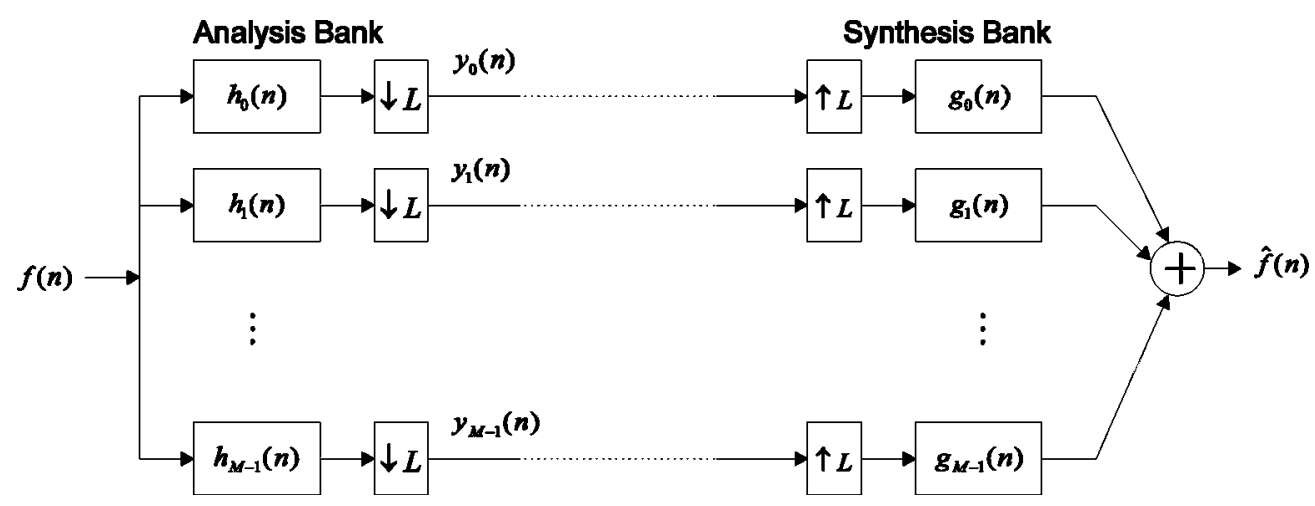

Fig. 1 Noise-free $M$-band filter-bank system.

The remainder of the paper is organized as follows. Section 2 provides the basic concept of filter-bank systems and the multichannel representation of decimators/interpolators. In Sec. 3 the 2-D multirate state-space model suitable for 2-D Kalman filtering is developed. The performance enhancement through object-plane partitioning is described in Sec. 4. Some numerical results and discussions are included in Sec. 5. Concluding remarks are provided in Sec. 6.

\section{Filter-Bank Systems and Problem Formulation}

\subsection{Filter Bank Systems}

The $M$-band filter-bank structure is shown in Fig. 1. The bank of filters $\left\{\mathbf{H}_{k}(z), k=0,1, \ldots, M-1\right\}$ comprises the analysis filters. Each filter output is downsampled and transmitted to the receiver, where it is upsampled and fed into the bank of synthesis filters $\left\{\mathbf{G}_{k}(z), k=1,2, \ldots, M-1\right\}$ for signal reconstruction. The decimation factor $L$ satisfies $L \leqslant M$. Without loss of generality, we consider only the maximal-decimation case, $L=M$, in this paper.

We can derive the transmission features of the filterbank systems from the block diagram shown in Fig. 1. If we were to remove the undesired aliasing distortion, we would have

$\hat{\mathbf{F}}(z)=\left[\frac{1}{M} \sum_{k=0}^{M-1} \mathbf{G}_{k}(z) \mathbf{H}_{k}(z)\right] \mathbf{F}(z)$.

The requirement for perfect reconstruction, i.e., $\hat{f}(n)$ $=f\left(n-n_{0}\right)$, is obviously

$\sum_{k=0}^{M-1} \mathbf{G}_{k}(z) \mathbf{H}_{k}(z)=z^{-n_{0}}$,

i.e., the composite transmission reduces to a simple delay.

\subsection{Multichannel Representation of Subband Signals}

Let $\left\{h_{i}, i=0,1, \ldots, M-1\right\}$ and $\left\{g_{i}, i=0,1, \ldots, M-1\right\}$ denote the impulse response of the bank of analysis filters $\left\{\mathbf{H}_{k}(z)\right.$, $k=0,1, \ldots, M-1\}$ and the bank of synthesis filters $\left\{\mathbf{G}_{k}(z)\right.$, $k=0,1, \ldots, M-1\}$, respectively. The $i$ th subband compo- nent $y_{i}$ is obtained by passing $f(n)$ through $h_{i}(n)$ and downsampling by $M$, which can be described as

$y_{i}(n)=\sum_{j} h_{i}(M n-j) f(j)$.

Let $j=M k+l, l=0,1, \ldots, M-1$, and substitute into Eq. (2.3). We have

$$
\begin{aligned}
y_{i}(n) & =\sum_{l=0}^{M-1} \sum_{k} h_{i}[M(n-k)-l] f(M k+l) \\
& =\sum_{l=0}^{M-1} \sum_{k} h_{i l}(n-k) f_{l}(k),
\end{aligned}
$$

where $h_{i l}(n)$ and $f_{l}(n)$ are the so-called polyphase components of $h_{i}(n)$ and $f(n)$, respectively, defined as

$h_{i l}(n)=h_{i}(M n-l), \quad l=0,1, \ldots, M-1$,

and

$f_{l}(n)=f(M n+l), \quad l=0,1, \ldots, M-1$.

Similarly, we have the reconstruction signal $\hat{f}(n)$ in terms of synthesis filters $g_{i}(n)$ and subband components $y_{i}(n)$ :

$\hat{f}(n)=\sum_{i=0}^{M-1} \sum_{k} g_{i}(n-M k) y_{i}(k)$.

Without loss of generality, we shall consider only the $M$ $=2$ case in the interest of convenient description; the derivations and results can be easily generalized to $M>2$ at the cost of notational complexity.

For $M=2$, we have the following equivalent multichannel representation of subband signals $y_{i}(n), i=0,1$, in view of (2.4):

$y(n)=\sum_{k=-\lambda}^{\Lambda} \mathbf{H}(k) \mathbf{f}(n-k)$,

where 


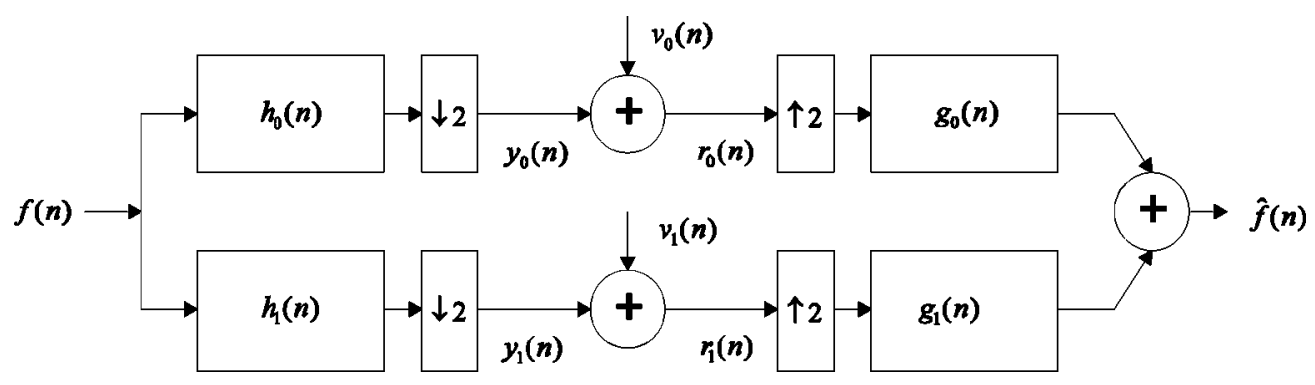

Fig. 2 Two-band filter-bank system with additive noise.

$\mathbf{f}(n)=\left[\begin{array}{c}f(2 n) \\ f(2 n+1)\end{array}\right]$ and $y(n)=\left[\begin{array}{l}y_{0}(n) \\ y_{1}(n)\end{array}\right]$

and $\mathbf{H}(k)$ is the multichannel impulse response matrix of the form

$\mathbf{H}(k)=\left[\begin{array}{ll}h_{00}(k) & h_{01}(k) \\ h_{10}(k) & h_{11}(k)\end{array}\right]$,

$h_{i 0}(k)=h_{i}(2 k)$ and $h_{i 1}(k)=h_{i}(2 k-1), i=0,1$.

If additive noisy corruptions are included in the subband components (see Fig. 2), the observed subband signal $\mathbf{r}(n)$ can be expressed as follows:

$\mathbf{r}(n)=\mathbf{y}(n)+\mathbf{v}(n)$.

Here $\mathbf{v}(n)=\left[v_{0}(n) \quad v_{1}(n)\right]^{\tau}$ is the additive noise disturbance vector. The conventional synthesis filters lose their optimality when an additive noisy disturbance $\mathbf{v}(n)$ is included in the subband components. We then seek to develop a multirate Kalman filter to replace the conventional synthesis bank $\left\{\mathbf{G}_{i}(z)\right\}_{i=0,1}$ for optimal signal reconstruction based on the observation $\mathbf{r}(n)$.

\section{Derivation of Multirate State-Space Model for 2-D Signal}

Subband image processing is the domain of extensive research. The idea of subband-based processing is to decompose the original image into different subbands and then employ separate processing in each subband. As we stated before, the overall performance of the 2-D perfectreconstruction filter bank deteriorates greatly when there is noisy corruption in subband components. Therefore, the optimal 2-D signal reconstruction from noisy subband systems remains a topic for research.

Figure 3 depicts the model of the image formation system, where the 2-D signal $f(i, j)$ (called the object-plane distribution in this paper) is assumed to be a wide-sense stationary Markov process and can be generated through a linear system $b(i, j)$ with a white source $u(i, j)$ as input. The 2-D signal $f(i, j)$ is then decomposed into its subband components $\mathbf{y}(i, j)$ through the 2-D filter bank system $\mathbf{H}(i, j)$. The final observation signal $\mathbf{r}(i, j)$ is the distorted version of $\mathbf{y}(i, j)$, which is corrupted with white noise $\mathbf{v}(i, j)$.

To incorporate the 2-D multirate Kalman filtering, a 2-D multirate state-space model, which represents the composite effects of the 2-D signal generating system $b(i, j)$, analysis filter bank $\mathbf{H}(i, j)$, and decimation operator, should be developed. The construction of the 2-D multirate statespace model involves the development of two state-space models: the vector dynamical model [known as $b(i, j)]$ and the basic signal model [known as $\mathbf{H}(i, j)]$. The two models are then combined to yield the final 2-D multirate statespace model suitable for 2-D Kalman filtering, which is used to replace the 2-D synthesis filter bank for optimal 2-D signal reconstruction from noisy subband systems.

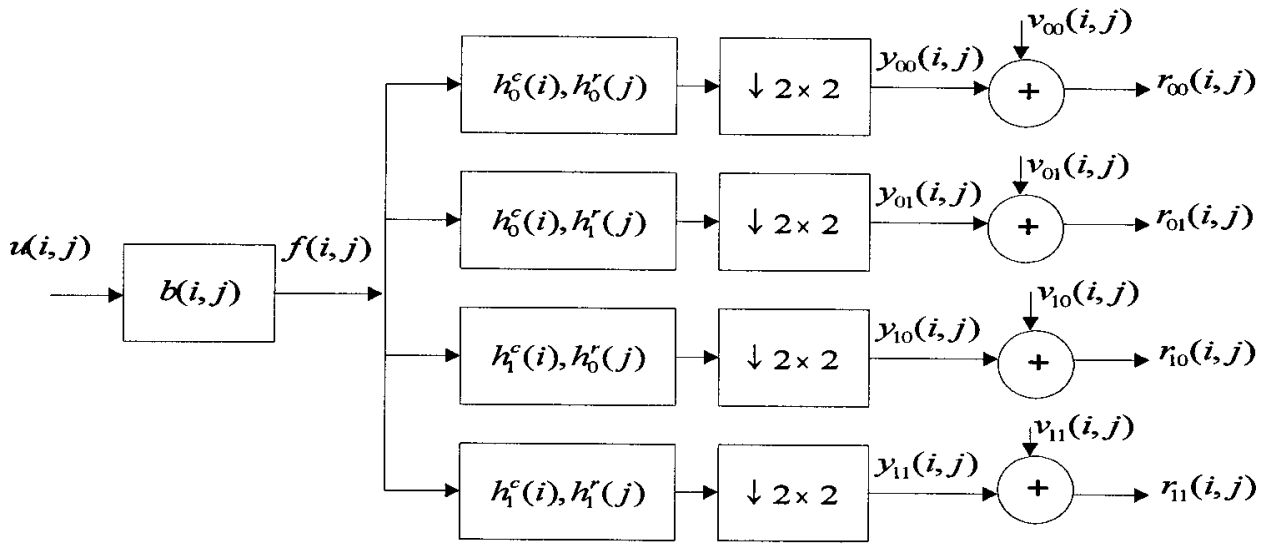

Fig. 3 Model of image formation system. 


\subsection{The Basic Signal Model}

When implementing the 2-D filter banks, the dominant approach is to apply two 1-D filter banks separately. Without loss of generality, we consider the $M=2$ case only, which is commonly used in practical applications. Let $\left\{h_{i}^{c}, g_{i}^{c}: i\right.$ $=0,1\}$ and $\left\{h_{i}^{r}, g_{i}^{r}: i=0,1\right\}$ denote two 1-D filter banks for column- and rowwise operations, respectively. The original 2-D signal $f(m, n)$ can be decomposed into four subband components $y_{i j}(m, n), i, j=0,1$, through the 2-D separate analysis banks

$y_{i j}(m, n)=\sum_{k} \sum_{l} h_{i}^{c}(l) h_{j}^{r}(k) f(2 m-l, 2 n-k)$

while the signal reconstruction can be expressed as

$\hat{f}(m, n)=\sum_{i=0}^{1} \sum_{j=0}^{1} \sum_{k} \sum_{l} g_{i}^{c}(m-2 l) g_{j}^{r}(n-2 k) y_{i j}(l, k)$.

Recalling the multichannel representation of the subband signal (2.7) described in the previous section, we can express Eq. (3.1) with the equivalent 2-D multichannel representation

$$
\begin{aligned}
\mathbf{y}(m, n) & =\left[\begin{array}{ll}
y_{00}(m, n) & y_{01}(m, n) \\
y_{10}(m, n) & y_{11}(m, n)
\end{array}\right] \\
& =\sum_{l=-\lambda^{c}} \sum_{k=-\lambda^{r}}^{\Lambda^{c}} \mathbf{H}^{c}(l) \mathbf{F}(m-l, n-k) \mathbf{H}^{r}(k)^{\tau}
\end{aligned}
$$

where

$\mathbf{F}(m, n)=\left[\begin{array}{cc}f(2 m, 2 n) & f(2 m, 2 n+1) \\ f(2 m+1,2 n) & f(2 m+1,2 n+1)\end{array}\right]$

and

$$
\begin{aligned}
& \mathbf{H}^{c}(k)=\left[\begin{array}{ll}
h_{00}^{c}(k) & h_{01}^{c}(k) \\
h_{10}^{c}(k) & h_{11}^{c}(k)
\end{array}\right], \\
& \mathbf{H}^{r}(k)=\left[\begin{array}{ll}
h_{00}^{r}(k) & h_{01}^{r}(k) \\
h_{10}^{r}(k) & h_{11}^{r}(k)
\end{array}\right] .
\end{aligned}
$$

Let $h_{i}^{c, r}$ be the column- or rowwise analysis filter, and $\left\{\lambda^{c, r}, \Lambda^{c, r}\right\}$ be the extent pair of $h_{i}^{c, r}$. The $\left\{\lambda^{c, r}, \Lambda^{c, r}\right\}$ in Eq. (3.3) are chosen such that $h_{i}^{c, r}\left(-2 \lambda^{c, r}-1\right)=0$ and $h_{i}^{c, r}\left(2 \Lambda^{c, r}+1\right)=0$.

By defining $p^{c}=\lambda^{c}+\Lambda^{c}$ and $p^{r}=\lambda^{r}+\Lambda^{r}$ and substituting $k=k-\lambda^{r}$ into Eq. (3.3), we can express $\mathbf{y}(m, n)$ as the causal form in the horizontal direction:

$\mathbf{y}(m, n)=\sum_{l=-\lambda^{c}}^{\Lambda^{c}} \sum_{k=0}^{p^{r}} \mathbf{H}^{c}(l) \mathbf{F}\left(m-l, n+\lambda^{r}-k\right) \mathbf{H}^{r}\left(k-\lambda^{r}\right)^{\tau}$.
If we define an operator $S_{l k}$ such that $S_{l k} \mathbf{A}=\mathbf{H}^{c}(l) \mathbf{A} \mathbf{H}^{r}(k$ $\left.-\lambda^{r}\right)^{\tau}$ and $D$ to be the horizontal shift operator with $D^{k} \mathbf{F}(m, n)=\mathbf{F}(m, n-k)$, the expression (3.4) can be transformed into the following form:

$$
\begin{aligned}
\mathbf{y}(m, n) & =\sum_{l=-\lambda^{c}}^{\Lambda^{c}} \sum_{k=0}^{p^{r}} S_{l k}\left[D^{k} \mathbf{F}\left(m-l, n+\lambda^{r}\right)\right] \\
& =\sum_{l=-\lambda^{c}}^{\Lambda^{c}} T_{l}(D) \mathbf{F}\left(m-l, n+\lambda^{r}\right)
\end{aligned}
$$

where $T_{l}(D)=S_{l 0}+S_{l 1} D+\cdots+S_{l p} D^{p^{r}}$.

We then expand Eq. (3.5) and arrange the elements according to the powers to $D$, i.e.,

$$
\begin{aligned}
\mathbf{y}(m, n)= & \sum_{l=-\lambda^{c}}^{\Lambda^{c}} S_{l 0} \mathbf{F}\left(m-l, n+\lambda^{r}\right)+\sum_{l=-\lambda^{c}}^{\Lambda^{c}} S_{l 1} D \mathbf{F}(m-l, n \\
& \left.+\lambda^{r}\right)+\cdots+\sum_{l=-\lambda^{c}}^{\Lambda^{c}} S_{l p^{r}} D^{p^{r}} \mathbf{F}\left(m-l, n+\lambda^{r}\right) .
\end{aligned}
$$

Let us further define a vector operator $\mathbf{S}_{k}$ and symbol $\overline{\mathbf{F}}(m, n)$ as follows:

$$
\begin{aligned}
\mathbf{S}_{k} \mathbf{A} & =\left[\begin{array}{llll}
\mathbf{H}^{c}\left(\Lambda^{c}\right) & \mathbf{H}^{c}\left(\Lambda^{c}-1\right) & \cdots & \mathbf{H}^{c}\left(-\lambda^{c}\right)
\end{array}\right] \mathbf{A} \mathbf{H}^{r}\left(k-\lambda^{r}\right)^{\tau} \\
& =\overline{\mathbf{H}}^{c} \mathbf{A} \mathbf{H}^{r}\left(k-\lambda^{r}\right)^{\tau},
\end{aligned}
$$

$\overline{\mathbf{F}}(m, n)=\left[\begin{array}{llll}\mathbf{F}\left(m-\Lambda^{c}, n\right) & \mathbf{F}\left(m-\Lambda^{c}+1, n\right) & \cdots & \mathbf{F}(m\end{array}\right.$

$$
\left.\left.+\lambda^{c}, n\right)\right]^{\tau} \text {. }
$$

Then Eq. (3.6) can be expressed in the compact form

$\mathbf{y}(m, n)=\sum_{k=0}^{p^{r}} \overline{\mathbf{H}}^{c}\left[D^{k} \overline{\mathbf{F}}\left(m, n+\lambda^{r}\right)\right] \mathbf{H}^{r}\left(k-\lambda^{r}\right)^{\tau}$.

We further represent Eq. (3.8) in a more convenient form with the powerful Kronecker product notation, namely,

$$
\begin{aligned}
\tilde{\mathbf{y}}(m, n) & =\sum_{k=0}^{p_{r}}\left[\mathbf{H}^{r}\left(k-\lambda^{r}\right) \otimes \overline{\mathbf{H}}^{c}\right]\left[D^{k} \tilde{\mathbf{F}}\left(m, n+\lambda^{r}\right)\right] \\
& =\sum_{k=0}^{p_{r}} \mathbf{C}_{k} D^{k} \tilde{\mathbf{F}}\left(m, n+\lambda^{r}\right)=\mathbf{H}(D) \tilde{\mathbf{F}}\left(m, n+\lambda^{r}\right),
\end{aligned}
$$

where $H(D)=\mathbf{C}_{0}+\mathbf{C}_{1} D+\cdots+\mathbf{C}_{p^{r}} D^{p^{r}}$. We obtain Eq. (3.9) from a property of Kronecker product notation, i.e., $\operatorname{vec}\{A B C\}=\left(C^{\tau} \otimes A\right) \operatorname{vec}\{B\},{ }^{6}$ where the operation $\tilde{A}$ $=\operatorname{vec}\{A\}$ means that the column vector $\widetilde{A}$ is formed by stacking up all columns of the matrix $A$. In view of the above definition, we have 
$\tilde{\mathbf{y}}(m, n)=\left[\begin{array}{llll}y_{00}(m, n) & y_{10}(m, n) & y_{01}(m, n) & y_{11}(m, n)\end{array}\right]^{\tau}$,

$$
\begin{aligned}
\tilde{\mathbf{F}}\left(m, n+\lambda^{r}\right)= & {\left[f [ 2 ( m - \Lambda ^ { c } ) , 2 ( n + \lambda ^ { r } ) ] \cdots f \left[2\left(m+\lambda^{c}\right)\right.\right.} \\
& \left.+1,2\left(n+\lambda^{r}\right)\right] f\left[2\left(m-\Lambda^{c}\right), 2\left(n+\lambda^{r}\right)+1\right] \\
& \left.\cdots f\left[2\left(m+\lambda^{c}\right)+1,2\left(n+\lambda^{r}\right)+1\right]\right]^{\tau} .
\end{aligned}
$$

The column vector $\tilde{\mathbf{F}}\left(m, n+\lambda^{r}\right)$ consists of two adjacent $2\left(p_{c}+1\right)$-dimensional column vectors $\mathbf{f}\left[m, 2\left(n+\lambda^{r}\right)\right]$ and $\mathbf{f}\left[m, 2\left(n+\lambda^{r}\right)+1\right]$ with vertical extent ranging from $2(m$ $\left.-\Lambda^{c}\right)$ to $2\left(m+\lambda^{c}\right)+1$.

The causal operator $H(D)$ in Eq. (3.9) can be equivalently described by a state-space model $(\mathbf{A}, \mathbf{B}, \mathbf{C}, \mathbf{D})$. If we define the state vector as

$$
\begin{aligned}
\mathbf{x}(m, n)= & {\left[\tilde{\overline{\mathbf{F}}}\left(m, n+\lambda^{r}-1\right)^{\tau} \tilde{\tilde{\mathbf{F}}}\left(m, n+\lambda^{r}-2\right)^{\tau} \cdots \tilde{\tilde{\mathbf{F}}}(m, n\right.} \\
& \left.\left.-\Lambda^{r}\right)^{\tau}\right]^{\tau},
\end{aligned}
$$

then we have

$\mathbf{x}(m, n+1)=\mathbf{A} \mathbf{x}(m, n)+\mathbf{B} \tilde{\mathbf{F}}\left(m, n+\lambda^{r}\right)$,

where

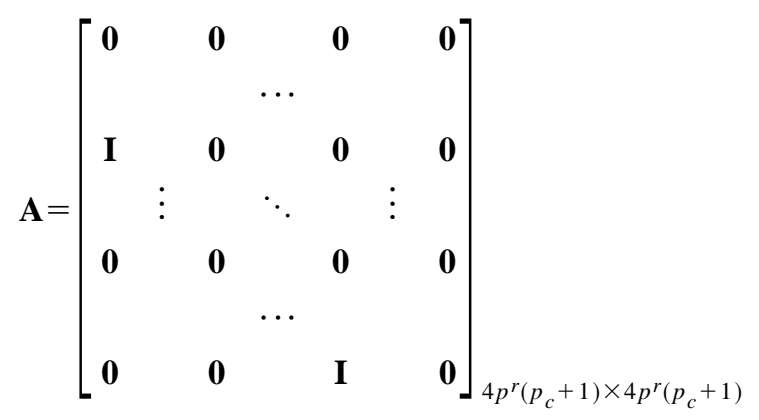

and

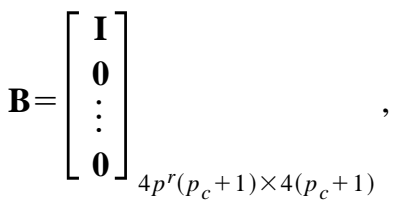

in which the $\mathbf{0}$ and $\mathbf{I}$ denote $4\left(p_{c}+1\right) \times 4\left(p_{c}+1\right)$ null and unit matrix, respectively.

We then expand Eq. (3.9) and notice that

$\mathbf{C}_{0}=\mathbf{H}^{r}\left(-\lambda^{r}\right) \otimes \overline{\mathbf{H}}^{c}=\left[\begin{array}{ll}h_{0}^{r}\left(-2 \lambda^{r}\right) \overline{\mathbf{H}}^{c} & \mathbf{0} \\ h_{1}^{r}\left(-2 \lambda^{r}\right) \overline{\mathbf{H}}^{c} & \mathbf{0}\end{array}\right]$.

Thus the subband output can be expressed as

$$
\begin{aligned}
\tilde{y}(m, n) & =\sum_{k=1}^{p_{r}} \mathbf{C}_{k} \tilde{\mathbf{F}}\left(m, n+\lambda^{r}-k\right)+\mathbf{C}_{0} \tilde{\mathbf{F}}\left(m, n+\lambda^{r}\right) \\
& =\mathbf{C x}(m, n)+\mathbf{D f}\left[m, 2\left(n+\lambda^{r}\right)\right]
\end{aligned}
$$

where

$$
\mathbf{C}=\left[\begin{array}{llll}
\mathbf{C}_{1} & \mathbf{C}_{2} & \cdots & \mathbf{C}_{p^{r}}
\end{array}\right]_{4 \times 4 p^{r}\left(p^{c}+1\right)}
$$

and

$\mathbf{D}=\left[\begin{array}{l}h_{0}^{r}\left(-2 \lambda^{r}\right) \overline{\mathbf{H}}^{c} \\ h_{1}^{r}\left(-2 \lambda^{r}\right) \overline{\mathbf{H}}^{c}\end{array}\right]_{4 \times 2\left(p^{c}+1\right)}$.

Based on these, we can formulate the 2-D noisy subband system as the following multirate state-space model:

$\mathbf{x}(m, n+1)=\mathbf{A x}(m, n)+\mathbf{B} \tilde{\mathbf{F}}\left(m, n+\lambda^{r}\right)$,

$\hat{\mathbf{x}}(m,-1)=\hat{\mathbf{x}}_{-1}$

$\widetilde{\mathbf{r}}(m, n)=\mathbf{C x}(m, n)+\mathbf{D f}\left[m, 2\left(n+\lambda^{r}\right)\right]+\widetilde{\mathbf{v}}(m, n)$

where $\tilde{\mathbf{r}}(m, n)=\left[r_{00}(m, n) r_{10}(m, n) r_{01}(m, n) r_{11}(m, n)\right]^{\tau}$ is the noisy observation vector and $\tilde{\mathbf{v}}(m, n)$ $=\left[v_{00}(m, n) v_{10}(m, n) v_{01}(m, n) v_{11}(m, n)\right]^{\tau}$ is the additive noise disturbance. To incorporate the 2-D multirate state-space model (3.12), we then seek to develop a vector dynamical model to represent the statistics of the input 2-D signal.

\subsection{The Vector Dynamical Model}

Consider the process $f(t, n)$. Its horizontal variable (continuous) is denoted by $t: 0 \leqslant t \leqslant T$, and its vertical variable by an integer $n=1,2, \ldots, N$. Without any loss of generality, $f(t, n)$ is chosen to have zero mean. The random process $f(t, n)$ is further assumed to be wide-sense stationary (WSSP) with autocorrelation function

$E\left[f\left(t_{1}, n_{1}\right) f\left(t_{2}, n_{2}\right)\right]=R\left(t_{2}-t_{1}, n_{2}-n_{1}\right)=R(t, n)$.

For many natural images the autocorrelation function $R(t, n)$ is well characterized by ${ }^{7}$

$R(t, n)=\alpha \exp \left(-\mu_{h}|t|-\mu_{v}|n|\right)$.

A vector dynamical model can also be developed that, when driven by a white vector noise, produces a vector output whose second-order statistics is described by Eq. (3.14). Let $\mathbf{x}(t)$ and $\mathbf{u}(t)$ be an $M$-dimensional state vector and a driving white noise vector, respectively, and $\mathbf{y}(t)$ be the $M$-dimensional output vector. Then the vector dynamical model can be described as

$\dot{\mathbf{x}}(t)=A \mathbf{x}(t)+B \mathbf{u}(t), \quad \mathbf{y}(t)=C \mathbf{x}(t)$,

where $A=-\mu_{h}$ and $B=\sqrt{2 \mu_{h}}$ are scalars, $C$ is an $M \times M$ matrix such that $C C^{\tau}=H$, and the $k, l$ th element $h_{k l}$ of $H$ is given by $h_{k l}=\alpha \exp \left(-\mu_{v}|k-l|\right)$. We consider the matrix correlation function of the output vector $\mathbf{y}(t)$, which is denoted by $\Theta(\eta)=E\left[\mathbf{y}(t) \mathbf{y}(t+\eta)^{\tau}\right]$. If $t$ and $t+\eta$ range over the domain $[0, T]$, then the $k, l$ th element $\theta_{k l}(\eta)$ of $\Theta(\eta)$ matches Eq. (3.14) exactly. The detailed procedure of parameter determination for the model (3.15) can be found in Ref. 5. 
We now complete the construction of the continuous vector dynamical model (3.15). Its discrete counterpart can be obtained through the following mapping ${ }^{7}$ :

$\bar{A}(k)=\exp (A T)$,

$\bar{B} \bar{K} \bar{B}^{\tau}=\int_{0}^{T} e^{A(T-s)} B K B^{\tau} e^{A^{\tau}(T-s)} \mathrm{d} s$,

$\bar{C}=C$,

where $K$ and $\bar{K}$ are the covariance matrices of $\mathbf{u}(t)$ and $\mathbf{u}(k)$, respectively. We then have the corresponding discrete vector model

$\mathbf{x}(k+1)=\bar{A} \mathbf{x}(k)+\bar{B} \mathbf{u}(k)$,

$y(k)=\bar{C} \mathbf{x}(k)$,

where

$E[\mathbf{u}(k)]=\mathbf{0}, \quad E\left[\mathbf{u}(k) \mathbf{u}(j)^{\tau}\right]=\mathbf{I} \delta(k-j)$,

$\mathbf{P}(-1)=E\left[\hat{\mathbf{x}}(-1) \hat{\mathbf{x}}(-1)^{\tau}\right], \quad \overline{\mathbf{C}} \mathbf{P}(-1) \overline{\mathbf{C}}^{\tau}=H$.

In view of the discussion above, the 2-D discrete vector model can be expressed in our configuration as

$\mathbf{z}^{\prime}(m, k+1)=\Phi \mathbf{z}^{\prime}(m, k)+\mathbf{G u}(m, k), \quad \hat{\mathbf{z}}^{\prime}(m,-1)=\hat{\mathbf{z}}_{m}^{\prime}$,

$\mathbf{f}(m, k)=\mathbf{H z}^{\prime}(m, k)$,

where $z^{\prime}(m, k)$ and $\mathbf{u}(m, k)$ are the $2\left(p_{c}+1\right)$-dimensional state vector and driving white-noise vector, respectively.

Examining the state-space model described by Eqs. (3.12) and (3.18) reveals that the two systems evolve on different time scales. We proceed to modify the model (3.18) so that the two models can be combined into an augmented model with the same time scale.

Substituting $k=2\left(n+\lambda^{r}\right)$ into Eq. (3.18) leads to

$$
\begin{aligned}
\mathbf{z}^{\prime}\left[m, 2\left(n+\lambda^{r}\right)+1\right]= & \Phi \mathbf{z}^{\prime}\left[m, 2\left(n+\lambda^{r}\right)\right] \\
& +\mathbf{G u}\left[m, 2\left(n+\lambda^{r}\right)\right],
\end{aligned}
$$

$\mathbf{f}\left[m, 2\left(n+\lambda^{r}\right)\right]=\mathbf{H z} \mathbf{z}^{\prime}\left[m, 2\left(n+\lambda^{r}\right)\right]$.

In the interest of developing a unified multirate state-space model, we further represent $\tilde{\bar{F}}\left(m, n+\lambda^{r}\right)$ and $\mathbf{f}[m, 2(n$ $\left.+\lambda^{r}\right)$ ] of Eq. (3.12) in terms of $\mathbf{z}^{\prime}(m, n)$ and $\mathbf{u}(m, n)$ :

$$
\begin{aligned}
\mathbf{z}^{\prime}\left[m, 2\left(n+\lambda^{r}\right)+2\right]= & \Phi^{2} \mathbf{z}^{\prime}\left[m, 2\left(n+\lambda^{r}\right)\right]+[\Phi \mathbf{G} \mathbf{G}] \\
& \times\left[\begin{array}{c}
\mathbf{u}\left[m, 2\left(n+\lambda^{r}\right)\right] \\
\mathbf{u}\left[m, 2\left(n+\lambda^{r}\right)+1\right]
\end{array}\right],
\end{aligned}
$$

$$
\begin{aligned}
\tilde{\mathbf{F}}\left(m, n+\lambda^{r}\right)= & {\left[\begin{array}{c}
\mathbf{f}\left[m, 2\left(n+\lambda^{r}\right)\right] \\
\mathbf{f}\left[m, 2\left(n+\lambda^{r}\right)+1\right]
\end{array}\right] } \\
= & {\left[\begin{array}{c}
\mathbf{H} \\
\mathbf{H} \Phi
\end{array}\right] \mathbf{z}^{\prime}\left[m, 2\left(n+\lambda^{r}\right)\right]+\left[\begin{array}{cc}
\mathbf{0} & \mathbf{0} \\
\mathbf{H G} & \mathbf{0}
\end{array}\right] } \\
& \times\left[\begin{array}{c}
\mathbf{u}\left[m, 2\left(n+\lambda^{r}\right)\right] \\
\mathbf{u}\left[m, 2\left(n+\lambda^{r}\right)+1\right]
\end{array}\right] .
\end{aligned}
$$

We define $\mathbf{z}(m, n)=\mathbf{z}^{\prime}\left[m, 2\left(n+\lambda^{r}\right)\right]$ to make the two systems (3.12) and (3.20) evolve on the same time scale, which leads to

$$
\begin{aligned}
& \mathbf{z}(m, n+1)=\Phi^{2} \mathbf{z}(m, n)+\mathbf{G}_{2} \mathbf{u}_{2}(m, n), \\
& \tilde{\mathbf{F}}\left(m, n+\lambda^{r}\right)=\mathbf{H}_{2} \mathbf{z}(m, n)+\mathbf{Q}_{2} \mathbf{u}_{2}(m, n), \\
& \mathbf{f}\left[m, 2\left(n+\lambda^{r}\right)\right]=\mathbf{H z}(m, n),
\end{aligned}
$$

where

$\mathbf{u}_{2}(m, n)=\left[\begin{array}{c}\mathbf{u}\left[m, 2\left(n+\lambda^{r}\right)\right] \\ \mathbf{u}\left[m, 2\left(n+\lambda^{r}\right)+1\right]\end{array}\right], \quad \mathbf{H}_{2}=\left[\begin{array}{c}\mathbf{H} \\ \mathbf{H} \Phi\end{array}\right]$,

$\mathbf{G}_{2}=\left[\begin{array}{ll}\Phi \mathbf{G} & \mathbf{G}\end{array}\right], \quad \mathbf{Q}_{2}=\left[\begin{array}{cc}\mathbf{0} & \mathbf{0} \\ \mathbf{H G} & \mathbf{0}\end{array}\right]$.

\subsection{The 2-D Multirate State-Space Model}

Under the assumption that the original 2-D signals (object plane) may be modeled as a zero-mean, two-dimensional, wide-sense stationary random field with known correlation function, we have derived a discrete vector model (3.21) to characterize the dynamics of the object plane. By defining $\mathbf{w}(m, n)=\left[\begin{array}{ll}\mathbf{x}(m, n)^{\tau} & \mathbf{z}(m, n)^{\tau}\end{array}\right]^{\tau}$ and substituting Eq. (3.21) for $\tilde{\mathbf{F}}\left(m, n+\lambda^{r}\right)$ and $\mathbf{f}\left[m, 2\left(n+\lambda^{r}\right)\right]$ in Eq. (3.12), we can obtain the final augmented 2-D multirate state-space model

$\mathbf{w}(m, n+1)=\overline{\mathbf{A}} \mathbf{w}(m, n)+\overline{\mathbf{B}} \mathbf{u}_{2}(m, n), \quad \hat{\mathbf{w}}(m,-1)=\hat{\mathbf{w}}_{m}$,

$\widetilde{\mathbf{r}}(m, n)=\overline{\mathbf{C}} \mathbf{w}(m, n)+\widetilde{\mathbf{v}}(m, n)$,

where $m=0,1, \ldots,(M / 2)-1$ and $n=0,1, \ldots,(N / 2)-1$ for an object plane with dimensions $M \times N$. The vector $\mathbf{w}(m, n)$ is defined as the state vector of the system (3.22) with dimension determined by that of $\mathbf{x}(m, n)$ and $\mathbf{z}(m, n)$. The parameters of the 2-D multirate state-space model are determined as follows:

$\overline{\mathbf{A}}=\left[\begin{array}{cc}\mathbf{A} & \mathbf{B H}_{2} \\ \mathbf{0} & \Phi^{2} \mathbf{I}\end{array}\right], \quad \overline{\mathbf{B}}=\left[\begin{array}{c}\mathbf{B Q}_{2} \\ \mathbf{G}_{2}\end{array}\right], \quad \overline{\mathbf{C}}=\left[\begin{array}{ll}\mathbf{C} & \mathbf{D H}\end{array}\right]$.

The 2-D multirate state-space model (3.22) reflects exactly the image formation model described in Fig. 3 with white driving source $\mathbf{u}(i, j)$ as input and $\mathbf{r}(i, j)$ as observation vector. The model allows us to take into account the dynamics of the object-plane distribution $f(i, j)$ in the process of optimal state estimation. 


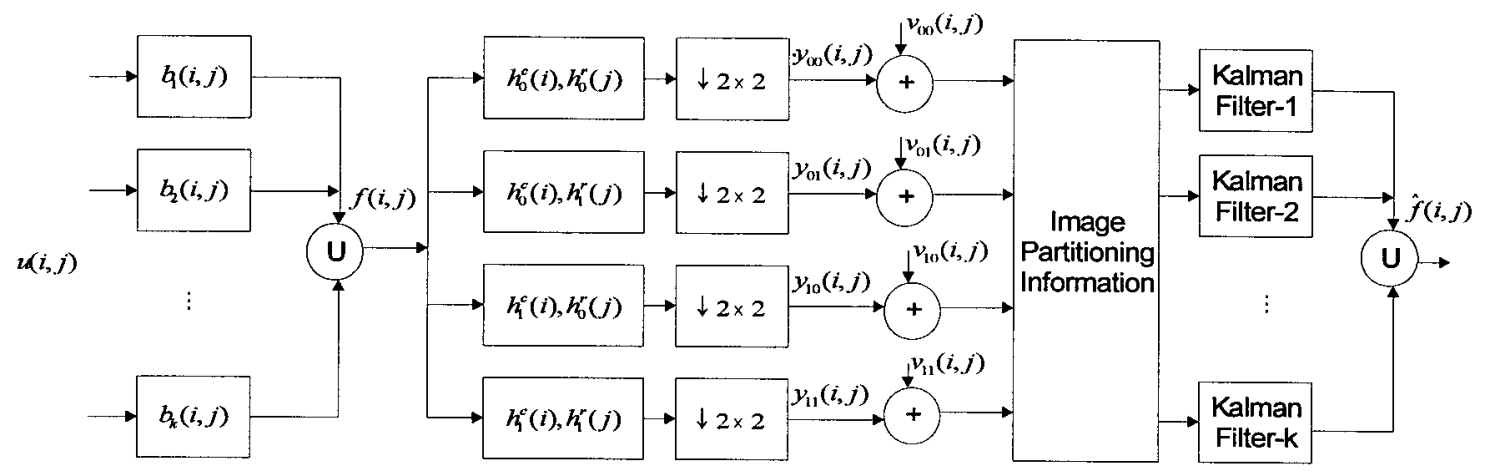

Fig. 4 General model of image formation system with adaptive 2-D multirate Kalman filters.

We then investigate the statistical characteristics of the driving source vector $\mathbf{u}_{2}(m, n)$ and noisy disturbance vector $\tilde{\mathbf{v}}(m, n)$ in the 2-D Kalman filtering configuration. The vector $\mathbf{u}_{2}(m, n)$ is formed by concatenation of two $2\left(p_{c}+1\right)$-dimensional driving vectors $\mathbf{u}\left[m, 2\left(n+\lambda^{r}\right)\right]$, $\mathbf{u}\left[m, 2\left(n+\lambda^{r}\right)+1\right]$. The second-order statistics of $\mathbf{u}_{2}(m, n)$ can be easily obtained from those of $\mathbf{u}\left[m, 2\left(n+\lambda^{r}\right)\right]$, $\mathbf{u}\left[m, 2\left(n+\lambda^{r}\right)+1\right]$, i.e.,

$E\left[\mathbf{u}_{2}(m, n)\right]=0$,

$E\left[\mathbf{u}_{2}(m, n) \mathbf{u}_{2}(m, k)^{\tau}\right]=\Omega(m, n) \delta(n-k)$

$$
\text { for } m=0,1, \ldots,(M / 2)-1 \text {. }
$$

The noisy disturbance vector $\widetilde{\mathbf{v}}(m, n)$ is also assumed to be a zero-mean, white Gaussian vector with covariance matrix of the form

$$
\begin{aligned}
E\left[\tilde{\mathbf{v}}(m, n) \tilde{\mathbf{v}}(m, k)^{\tau}\right] & =\left[\begin{array}{lll}
\sigma_{v_{0}}^{2}(m, n) & & \\
& \ddots & \\
& & \sigma_{v_{3}}^{2}(m, n)
\end{array}\right] \\
& =\mathbf{R}(m, n) \delta(n-k) .
\end{aligned}
$$

The Kalman synthesis filter equations for the 2-D subband system are given below for the sake of completeness:

$$
\hat{\mathbf{w}}(m, n)=[\mathbf{I}-\mathbf{K}(m, n) \overline{\mathbf{C}}] \overline{\mathbf{A}} \hat{\mathbf{w}}(m, n-1)+\mathbf{K}(m, n) \tilde{\mathbf{r}}(m, n),
$$

$$
\begin{aligned}
& \mathbf{K}(m, n)=\mathbf{P}(m, n) \overline{\mathbf{C}}^{\tau}\left[\overline{\mathbf{C}} \mathbf{P}(m, n) \overline{\mathbf{C}}^{\tau}+\mathbf{R}(m, n)\right]^{-1}, \\
& \mathbf{P}(m, n+1)=\overline{\mathbf{A}}[\mathbf{I}-\mathbf{K}(m, n) \overline{\mathbf{C}}] \mathbf{P}(m, n) \overline{\mathbf{A}}^{\tau}+\overline{\mathbf{B}} \Omega(m, n) \overline{\mathbf{B}}^{\tau},
\end{aligned}
$$

with $\quad \hat{\mathbf{w}}(m,-1)=\hat{\mathbf{w}}_{m}, \quad \mathbf{P}(m,-1)=\mathbf{P}_{m} \quad$ for $\quad m=0$, $1, \ldots,(M / 2)-1$ and $n=0,1, \ldots,(N / 2)-1$. The $\mathbf{P}(m, n)$ and $\mathbf{K}(m, n)$ in Eq. (3.26) are the filtering state error covariance matrices and the Kalman-filter gain, respectively.

\section{Performance Enhancement through Adaptive Kalman Filtering}

Recall the assumption that the 2-D signal $f(i, j)$ is characterized by a single wide-sense stationary process, whose autocorrelation function is of the form (3.14). This may not always be the case and can in fact lead to undesirable results. Considering the situation when there exists a large spread of spatial activity content in the object-plane distribution $f(i, j)$, the two parameters $\mu_{h}$ and $\mu_{v}$ in Eq. (3.14) may vary considerably from region to region.

The dependence of the autocorrelation function on spatial activity can be used in partitioning the object plane $f(i, j)$ and designing the corresponding multirate Kalman filter for each partition. Figure 4 shows the general model of an image formation system with adaptive multirate Kalman filtering. The original 2-D signal $f(i, j)$ is modeled as a set of white sources passed through a bank of linear generating systems, whose impulse responses are $b_{p}(i, j), p$ $=1,2, \ldots, k$. Consequently, the object plane $f(i, j)$ is composed of a set of disjoint partitioned regions $\Omega_{p}$. Each of these regions is assumed to be a 2-D stationary random process with a known autocorrelation function of the form (3.14), i.e.,

$R_{p}(t, n)=\alpha_{p} \exp \left(-\mu_{p, h}|t|-\mu_{p, v}|n|\right), \quad p=1,2, \ldots, k$.

A different multirate Kalman filter is designed for each of the partitioned regions based on its autocorrelation function $R_{p}(t, n)$ and the basic signal model (3.12) for optimal 2-D signal reconstruction from noisy subband components in an adaptive mode. The adaptive multirate Kalman filter can be viewed as a collection of $k$ separate multirate Kalman filters with a selection rule that determines which of the $k$ filters is used for signal reconstruction, based on the observation vector $\mathbf{r}(i, j)$.

The two key issues involved in the design of a 2-D adaptive multirate Kalman filter are (1) the partitioning of the object plane $f(i, j)$ based on the spatial activity content, and (2) the construction of a multirate Kalman filter for each partition $\Omega_{p}$.

There are a number of ways in which the object plane $f(i, j)$ can be partitioned. One simple and feasible approach 
is the so-called block partitioning on the basis of the variance within the block. The partitioning scheme is given as follows:

1. The object plane $f$ is divided into blocks of size $d$ $\times d$, resulting in the block sequence $\{M(m, n)\}$.

2. The variance of each block $M(m, n)$ is computed, which is defined as $\operatorname{Var}(m, n)=\sum_{(i, j) \in M(m, n)}[f(i, j)$ $-\eta(m, n)]^{2}$, where $\eta(m, n)$ is the mean for block $M(m, n)$.

3. A threshold operation is applied to segment the variance $\operatorname{Var}(m, n)$ so that the object plane $f$ is partitioned into regions $\Omega_{p}, p=1,2, \ldots, k$. The block $M(m, n)$ is classified as region $\Omega_{p}$ if $a_{p-1}$ $<\operatorname{Var}(m, n)<a_{p}$, where the threshold value $a_{p}$ is chosen subjectively according to the range of $\operatorname{Var}(m, n)$ and the number of regions desired.

We then proceed to develop the 2-D multirate statespace model for the region $\Omega_{p}$. Referring to the general signal formation model described in Fig. 4, the linear generating system $b_{p}(i, j)$ can be represented equivalently by a vector dynamical model of the form (4.2):

$\mathbf{z}_{p}(m, n+1)=\Phi_{p}^{2} \mathbf{z}_{p}(m, n)+\mathbf{G}_{p, 2} \mathbf{u}_{p, 2}(m, n)$,

$\tilde{\mathbf{F}}\left(m, n+\lambda^{r}\right)=\mathbf{H}_{p, 2} \mathbf{z}_{p}(m, n)+\mathbf{Q}_{p, 2} \mathbf{u}_{p, 2}(m, n)$,

$\mathbf{f}\left[m, 2\left(n+\lambda^{r}\right)\right]=\mathbf{H}_{p} \mathbf{z}_{p}(m, n)$.

The model (4.2) is constructed specifically for the region $\Omega_{p}$ and has the similar form as (3.21). The model (4.2) can then be combined with the basic signal model (3.12) to yield the multirate statespace model (4.3) for the region $\Omega_{p}$ :

$\mathbf{w}_{p}(m, n+1)=\overline{\mathbf{A}}_{p} \mathbf{w}_{p}(m, n)+\overline{\mathbf{B}}_{p} \mathbf{u}_{p, 2}(m, n)$,

$\widetilde{\mathbf{r}}(m, n)=\overline{\mathbf{C}}_{p} \mathbf{w}_{p}(m, n)+\widetilde{\mathbf{v}}(m, n), \quad p=1,2, \ldots, k$.

On the basis of the model $\left(\overline{\mathbf{A}}_{p}, \overline{\mathbf{B}}_{p}, \overline{\mathbf{C}}_{p}\right)$, a set of multirate Kalman filters are constructed to provide the optimal estimate of the original 2-D signal $f(i, j)$ restricted to region $\Omega_{p}$.

\section{Numerical Results and Discussions}

Computer simulations were carried out to show the feasibility and effectiveness of the proposed 2-D multirate Kalman filtering for optimal 2-D signal reconstruction from noisy subband systems. We compared the performance of the proposed 2-D multirate Kalman filter with that of the conventional PR filter banks for 2-D signal reconstruction at different noise levels. The simulation was implemented on the SGI/R4000 platform with Matlab and C.

We adopted a set of two-band QMF PR filter banks of length 8 in our simulation, which is defined as $h(n)=\left[\begin{array}{lllllll}0.0094 & -0.0707 & 0.0694 & 0.4900 & 0.4900 & 0.0694\end{array}\right.$ $-0.07070 .0094]$, with $n$ ranging from -4 to 3 . The analysis filter bank is defined by $h_{0}(n)=h(n)$ and $h_{1}(n)$ $=(-1)^{n} h(n)$, while the corresponding synthesis filter bank is given by $g_{0}(n)=2 h(n)$ and $g_{1}(n)=-2(-1)^{n} h(n)$. The $2 \times 2$ subband decomposition of test image is implemented through two 1-D separate analysis filter banks $\left\{h_{i}^{c}, h_{i}^{r}\right\}_{i=0,1}$, with $h_{i}^{c}(n)=h_{i}^{r}(n)=h_{i}(n), i=0,1$.

Two quantitative measures are defined in our simulation to reflect the input noise level and reconstruction performance. We define

$\mathrm{SNR}_{i}^{m, n}=10 \log _{10}\left[\frac{\sum_{k, l} y^{m, n}(k, l)^{2}}{\sum_{k, l} v^{m, n}(k, l)^{2}}\right], \quad m, n=0,1$,

where $y^{m, n}(k, l)$ and $v^{m, n}(k, l)$ are the component and noise in subband $(m, n)$, respectively. We denote $\mathrm{SNR}_{i}^{m, n}$ as $\mathrm{SNR}_{i}$, since we adopted the same SNR level in each subband in our simulation. Another measure, $\mathrm{SNR}_{r}$, is used to indicate the reconstruction performance and defined as

$\mathrm{SNR}_{r}=10 \log _{10}\left\{\frac{\sum_{k, l} f(k, l)^{2}}{\sum_{k, l}[f(k, l)-\hat{f}(k, l)]^{2}}\right\}$,

where $f(k, l)$ and $\hat{f}(k, l)$ denote the original and reconstructed 2-D signal, respectively.

Case 1: Natural image with fixed model. The test image "Hillside" is of size $160 \times 160 \times 8$ [Fig. 5(a)]. The image is converted to zero mean prior to $2 \times 2$ subband decomposition. Its autocorrelation function is obtained from the entire image without partitioning and found to have the form $R(t, n)=1168.3 \exp (-0.15|t|-0.22|n|)$. The vertical extent of $h_{i}^{c}$ is $2\left(p^{c}+1\right)=10$ for the given analysis filter bank. A 10-dimensional vector dynamical model of the image is constructed on the basis of its $R(t, n)$. $^{5}$

Figure 5(b) shows the $2 \times 2$ subband decomposition of the image "Hillside." White additive noise at different SNR levels was added to all the four subband images. The variance of the white noise was adjusted so that the SNR level on each subband image was kept the same. Figure 6 demonstrates the reconstruction performance comparison with both the proposed 2-D multirate Kalman filtering and the conventional PR synthesis filters at different SNR levels. Figure 5(d) shows the noise-corrupted subband image at $\mathrm{SNR}_{i}=0 \mathrm{~dB}$. Figures 5(e) and 5(f) represent the reconstructed images at $\mathrm{SNR}_{i}=0 \mathrm{~dB}$ with the conventional filterbank and the Kalman filtering approach, while Figs. 5(g) and $5(\mathrm{~h})$ depict the results at $\mathrm{SNR}_{i}=8 \mathrm{~dB}$. It is observed that the improvement in reconstruction SNR with the proposed 2-D multirate Kalman filtering is also considerable, especially in the portion of low $\mathrm{SNR}_{i}$. The noise effects are well suppressed, even at extremely low SNR [Fig. 5(f)]: the main structure of original image is still distinguishable on the reconstructed image with 2-D multirate Kalman filtering.

Case 2: Natural image with adaptive model. The test image "Hillside" was partitioned into two regions based on their variance distributions. The segmented image is dis- 


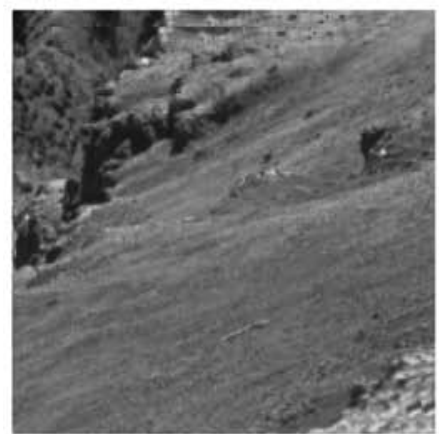

(a)

Original image

Hillside of size 160x160

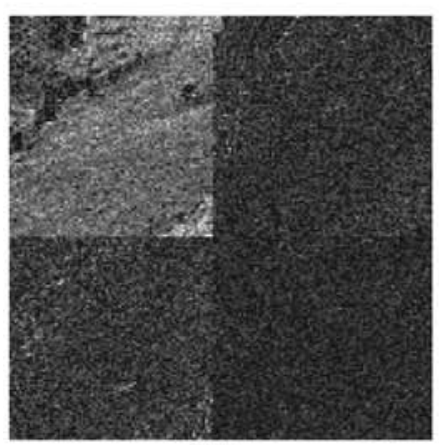

(d)

Noise-corrupted subband image at $S N R_{i}=0 d b$

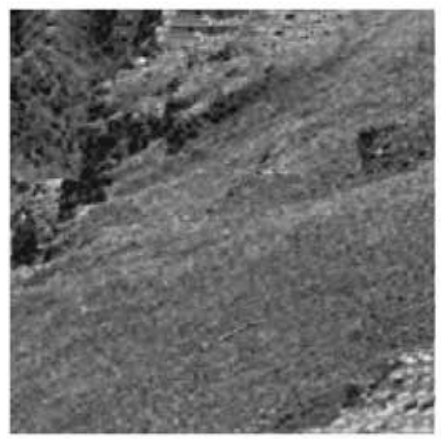

(g)

Reconstruction image with conventional PR filter banks at $S N R_{i}=8 d b$

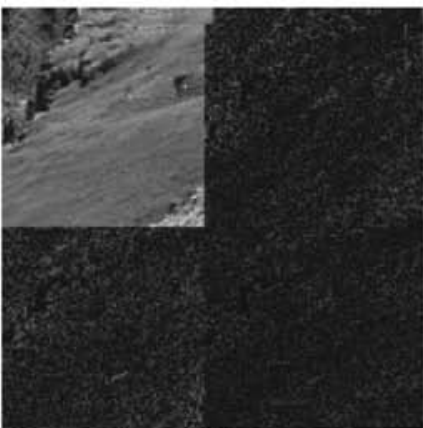

(b)

$2 \times 2$ subband decomposition of Hillside

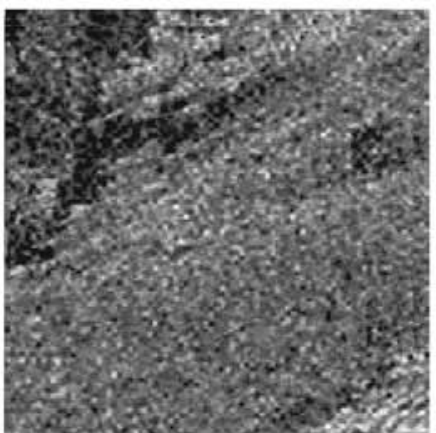

(e)

Reconstruction image with conventional PR filter banks at $S N R_{i}=0 d b$

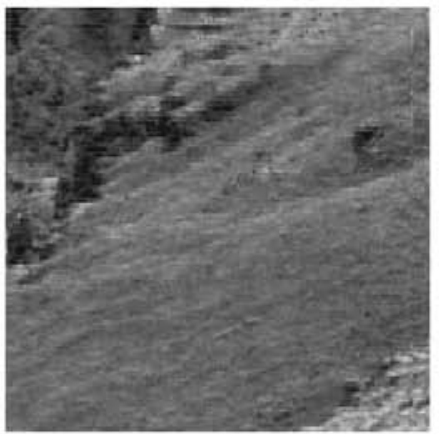

(h)

Reconstruction image with 2-D fixed-model multirate Kalman filter at $S N R_{i}=8 d b$

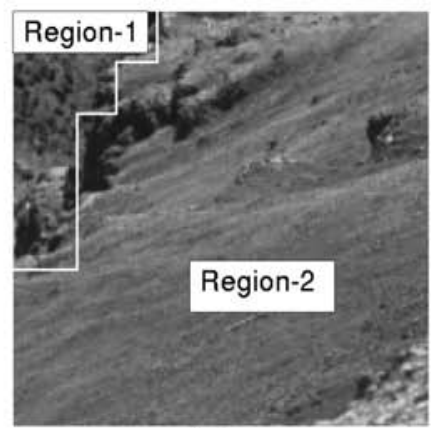

(c)

partitioned image

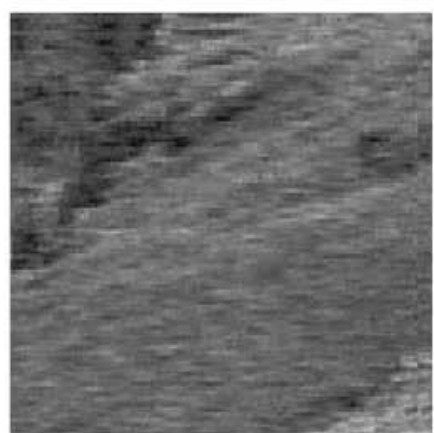

(f)

Reconstruction image with 2-D multirate Kalman

filter at $S N R_{i}=0 \mathrm{db}$

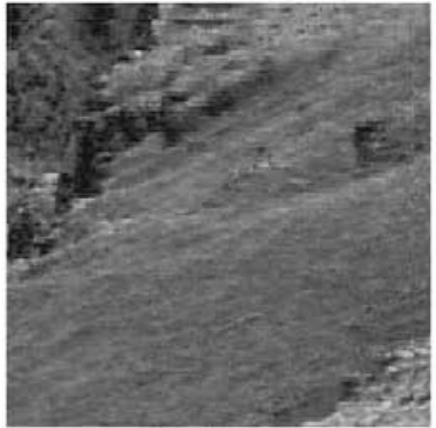

(i)

Reconstruction image with 2-D adaptive multirate Kalman

filter at $S N R_{i}=8 d b$

Fig. 5 (a) Original image "Hillside" of size $160 \times 160$. (b) $2 \times 2$ subband decomposition of "Hillside." (c) Partitioned image. (d) Noise-corrupted subband image at $\mathrm{SNR}_{i}=0 \mathrm{~dB}$. (e) Reconstructed image with conventional PR filter banks at $\mathrm{SNR}_{i}=0 \mathrm{~dB}$. (f) Reconstructed image with 2-D multirate Kalman filter at $\mathrm{SNR}_{i}=0 \mathrm{~dB}$. (g) Reconstructed image with conventional PR filter banks at $\mathrm{SNR}_{i}=8 \mathrm{~dB}$. (h) Reconstructed image with 2-D fixed-model multirate Kalman filter at $\mathrm{SNR}_{i}=8 \mathrm{~dB}$. (i) Reconstructed image with 2-D adaptive multirate Kalman filter at $\mathrm{SNR}_{i}=8 \mathrm{~dB}$. 


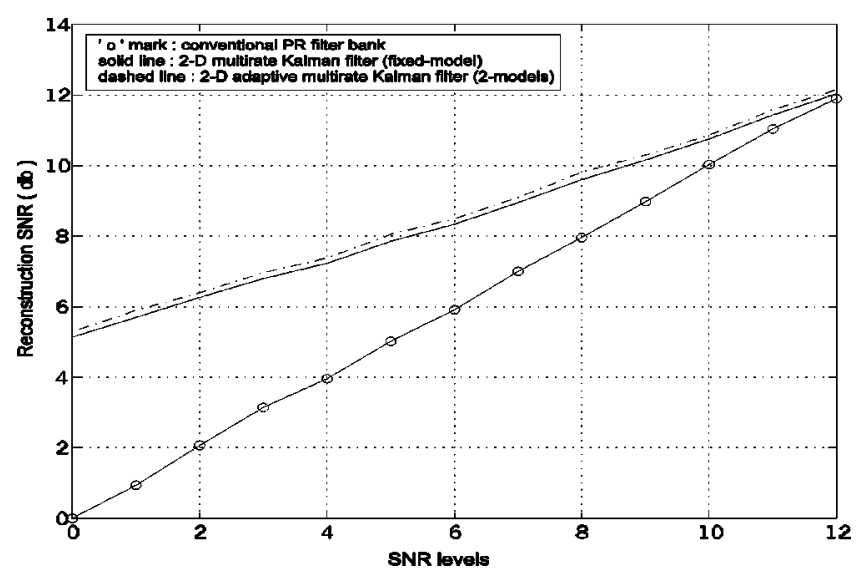

Fig. 6 Reconstruction SNR versus additive-noise SNR levels for natural image "Hillside" of size $160 \times 160$.

played in Fig. 5(c), where the rock texture in the upper left corner is classified as Region 1 and the rest of the image as Region 2. The two autocorrelation functions are then determined for each region and have the form

region $\Omega_{1}: \quad R_{1}(t, n)=2548.6 \exp (-0.12|t|-0.16|n|)$;

region $\Omega_{2}: \quad R_{2}(t, n)=849.8 \exp (-0.17|t|-0.27|n|)$.

From these autocorrelation functions the appropriate values are determined for the matrices $\left\{\overline{\mathbf{A}}_{p}, \overline{\mathbf{B}}_{p}, \overline{\mathbf{C}}_{p}\right\}_{p=0,1}$, which are used for signal reconstruction in region 1 and region 2 , respectively. Figure 4 shows the block diagram for this adaptive Kalman filtering scheme. The result of application of the adaptive Kalman filtering at $\mathrm{SNR}_{i}=8 \mathrm{~dB}$ is shown in Fig. 5(i), with the result of fixed-model Kalman filtering for comparison. The reconstructed image with the two-model adaptive Kalman filtering approach is observed to have better appearance. The edges tend to be somewhat sharper, and the texture of the rocks in the upper left corner is more distinct. The performance comparison between fixed-model and adaptive-model multirate Kalman filtering at different SNR levels is also given in Fig. 6. The SNR improvement with the adaptive approach for the "Hillside" image is around $0.2 \mathrm{~dB}$. Although we are not here with refining the adaptive algorithm, the preliminary results with the adaptive Kalman filtering approach show potential to further improve the dynamical performance of the proposed 2-D multirate Kalman filter.

\section{Conclusions}

In this paper, we have combined the multichannel representation of subband signal with the statistical model of input signals to derive the multirate state-space model for noisy filter-bank systems, with emphasis on the 2-D case. With the input signals embedded in the state vector, the issue of signal reconstruction can be formulated as optimal state estimation with multirate Kalman filtering. Since the Kalman filtering is one-dimensional in nature, the vector dynamical model is incorporated to develop a 2-D multirate state-space model suitable for 2-D multirate Kalman filtering.
Computer simulation results with a natural image demonstrate considerable improvements in the $\mathrm{SNR}_{r}$ of the reconstructed 2-D signal, at various noise levels, with the proposed 2-D multirate Kalman filtering approach over those with conventional PR synthesis filters.

We further present a 2-D adaptive multirate Kalman filtering scheme based on image segmentation. The partitioning of the image allows more accurate modeling of the second-order statistics of the object-plane distribution function and provides the potential for further performance enhancement.

\section{References}

1. P. P. Vaidyanathan, "Multirate digital filters, filter banks, polyphase networks, and applications: a tutorial," Proc. IEEE 78(1), 56-93 (1990).

2. J. Kovacevic, "Subband coding systems incorporating quantizer models," IEEE Trans. Image Process. 4(5), 543-553 (1995).

3. A. N. Delopoulos and S. D. Kollias, "Optimal filter banks for signal reconstruction from noisy subband components," IEEE Trans. Signal Process. 44(2), 212-224 (1996).

4. B. S. Chen, C. W. Lin, and Y. L. Chen, "Optimal signal reconstruction in noisy filter bank systems: multirate Kalman synthesis filtering approach," IEEE Trans. Signal Process. 43(11), 2496-2504 (1995).

5. N. E. Nahi, "Recursive image enhancement-vector processing," IEEE Trans. Comm. 21, 305-311 (1973).

6. J. M. Ortega, Matrix Theory, A Second Course, Plenum, New York (1987).

7. L. E. Franks, “A model for a random video process,'” Bell Syst. Tech. J. 45, 609-630 (1966).

8. N. E. Nahi, Estimation Theory and Applications, Wiley, New York (1969).

9. J. Q. Ni, K. L. Ho, and K. W. Tse, "Multirate Kalman filtering approach for optimal two-dimensional signal reconstruction from noisy subband systems," in Proc. 1997 Int. Conf. on Image Processing, ICIP'97, IEEE Signal Processing Society, Santa Barbara (1997).

10. A. O. Aboutlib, "Digital restoration of images degraded by general motion blurs,"' IEEE Trans. Automat. Control AC-22(3), 294-302 (1977).

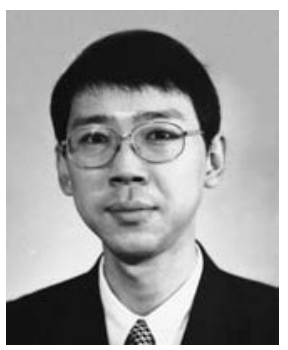

Jiang Qun Ni received BS and MS degrees from Wuhan University of Hydraulic and Electric Engineering, Wuhan, China, in electronic engineering in 1985 and 1988 , respectively. He was a lecturer at the same university during 1988-1993. Since 1994, he has been with the Department of Electrical and Electronic Engineering at the University of Hong Kong, where he is currently a graduate research assistant and working toward the PhD degree. His research interests include filter banks, wavelets, and image and video coding.

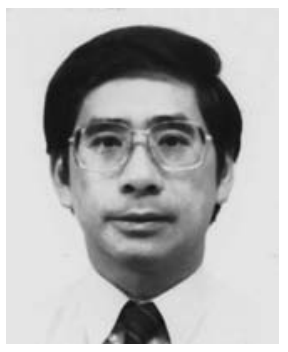

Ka Leung Ho received the $\mathrm{BSc}(\mathrm{Eng})$ and the MPhil. degrees in electronic engineering from the University of Hong Kong in 1971 and 1973, respectively, and the PhD degree from the University of London in 1977. In 1984, he joined the Department of Electrical and Electronic Engineering at the University of Hong Kong. His current research interests include wave propagation, signal processing, and communication systems. $\mathrm{He}$ is a chartered engineer of the Engineering Council, UK, and a member of the IEE, HKIE, and IEEE. 
$\mathrm{Ni}$, Ho, and Tse: Model-based multirate Kalman filtering approach ...

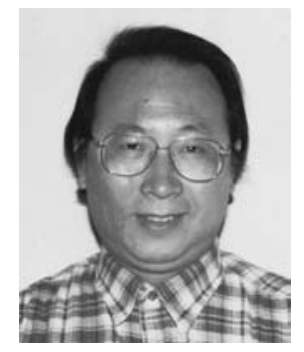

Kai Wing Tse received the BSc (Hons) and $\mathrm{PhD}$ degrees from the University of Manchester, UK, in 1977 and 1986 respectively. He had about nine years of industrial experience at Burroughs $\mathrm{Ma}$ chines Ltd. (UK), ICL (UK), Bell-Northern Research (Canada), and Microtel Pacific Research (Canada) from 1977 to 1986. He has been a lecturer in the Department of Electrical and Electronic Engineering, University of Hong Kong, since 1986. His research interests are in communication, networks, and computer architecture and applications. 